\title{
Synergistic Microgel-Reinforced Hydrogels as High-Performance Lubricants
}

\author{
Jing $\mathrm{Hu}$, Efren Andablo-Reyes, Siavash Soltanahmadi, and Anwesha Sarkar*
}

Cite This: ACS Macro Lett. 2020, 9, 1726-1731

Read Online

ABSTRACT: The ability to create a superlubricious aqueous lubricant is important for various biological and technological applications. Here, a nonlipid biolubricant with strikingly low friction coefficients is fabricated (patented) by reinforcing a fluidlike hydrogel composed of biopolymeric nanofibrils with proteinaceous microgels, which synergistically provide superlubricity on elastomeric surfaces in comparison to any of the sole components.

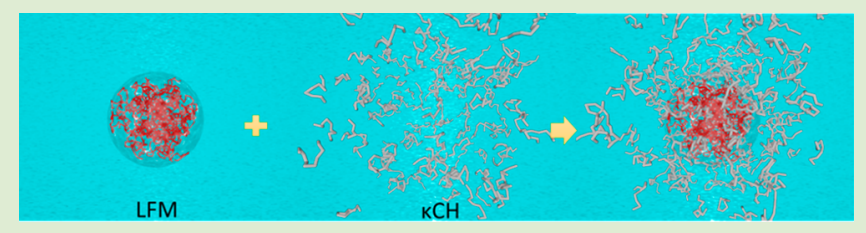
This two-component lubricant composed of positively charged lactoferrin microgels and negatively charged $\kappa$-carrageenan hydrogels is capable of exceeding the high lubricating performance of real human saliva in tribo tests using both smooth and textured surfaces, latter mimicking the human tongue's wettability, topography, and compliance. The favorable electrostatic attraction between mutually oppositely charged microgels and the hydrogel reinforces the mechanical properties of the hydrogel, allowing friction reduction by combining the benefits of both viscous and hydration lubrication. The superlubricity of these microgel-reinforced hydrogels offers a unique prospect for the fabrication of biocompatible aqueous lubricants for dry-mouth therapy and/or designing of nonobesogenic nutritional technologies.

$\mathrm{W}$ ater forms the basis of all biological lubrication systems such as tears, saliva, and synovial fluids in humans. ${ }^{1}$ Hydrogels and microgels are both composed of cross-linked water-swollen polymer networks, with the latter in the form of micron-sized particles. They have been extensively used to improve the rheological ${ }^{2,3}$ and mechanical properties of biomaterials and other technological applications. ${ }^{4-6}$ Recently, biopolymeric microgels have been reported as effective lubricants for elastomeric surfaces due to their capacity to trap water molecules, providing hydration lubrication. ${ }^{2,7}$ Here, for the first time, we demonstrate that reinforcing a carbohydrate hydrogel with proteinaceous microgels can result in superlubricity on polydimethylsiloxane (PDMS), a commonly used material for investigation of oral processes and a novel 3D tongue-like biomimicking silicon surface. The twocomponent lubricant is composed of positively charged lactoferrin microgels (LFMs) dispersed in negatively charged $\kappa$-carrageenan hydrogels $(\kappa \mathrm{CHs})$. The synergistic effect between the components enhances the hydration lubrication, resulting in significantly lower friction coefficient values in comparison to the sole components. This patented lubricant formulation $^{8}$ is capable of exceeding the lubricity of real human saliva in orally relevant tribo-contact conditions.

All solutions are made in 4-(2-hydroxyethyl)-1-piperazineethanesulfonic acid (HEPES buffer) at $\mathrm{pH}$ 7.0. LFMs are fabricated by thermal gelation of lactoferrin protein solution followed by passing through a jet homogenizer as described in the Supporting Information, Experimental Section. Size and morphological characteristics of LFM are shown in Figure 1a. The size distribution of LFM, obtained using dynamic light scattering (DLS), is unimodal with an average hydrodynamic diameter $\left(d_{\mathrm{H}}\right)$ of $155.0 \mathrm{~nm}$ and a polydispersity index of 0.2 . The transmission electron microscopy (TEM) image of LFMs confirms well-dispersed spheres corroborating with the DLS data. $\kappa \mathrm{CH}$ is prepared by dissolving $\kappa$-carrageenan in the buffer under heating and shearing conditions. In Figure $1 b$, a TEM image of $\kappa \mathrm{CH}$ shows typical bundles of nanofibril-shaped $\kappa \mathrm{Cnf}$ ( $\kappa$-carrageenan nanofibrils). ${ }^{9}$ These $\kappa \mathrm{Cnf}$ form an interconnected network with random distribution and orientation interacting via hydrogen bonds, resulting in gel-like structures with macroscale viscoelastic properties. ${ }^{10}$ LFM-reinforced $\kappa \mathrm{CHs}$ are fabricated by dispersing LFMs in $\kappa \mathrm{CH}$ at the ratio of 0.07:1 wt./vol. Figure $1 c_{1}$ clearly shows LFM particles dispersed in the interconnected network of the $\kappa$ Cnf-assisted hydrogel. Figure $1 c_{2}$ is the magnified image of LFMs embedded in $\kappa \mathrm{CH}$, indicating coverage of the LFMs by $\kappa \mathrm{Cnf}$.

The interaction between LFM particles and $\kappa \mathrm{Cnf}$ is expected to produce a synergistic effect towards the lubrication capacity of the LFM-reinforced $\kappa \mathrm{CH}$. Based on these TEM images, a conceptual representation of the system is presented in Figure 1d, where a spherical cross-linked proteinaceous network represents the microgel and grey-colored rod-like flexible

Received: September 22, 2020

Accepted: November 10, 2020

Published: November 16, 2020 


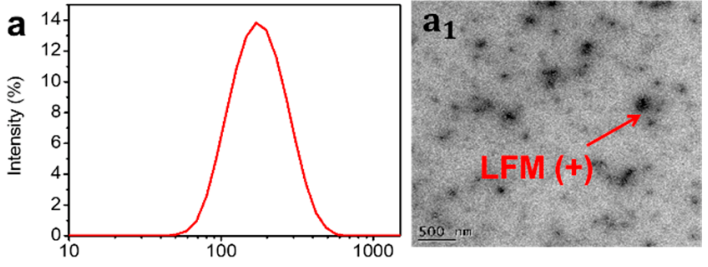

Hydrodynamic diameter $(\mathrm{nm})$
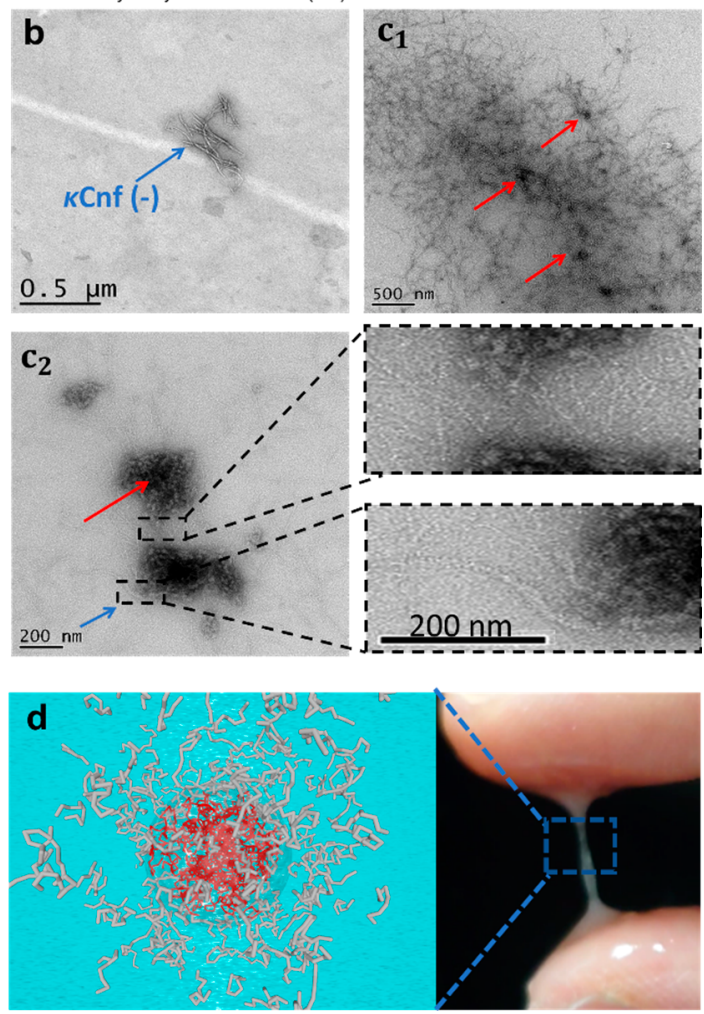

Figure 1. Mesoscopic structure of the lubricants. (a) Particle size distribution of LFMs obtained by dynamic light scattering showing a monomodal peak with hydrodynamic diameter $\left(d_{\mathrm{H}}\right) \sim 150 \mathrm{~nm} .\left(\mathrm{a}_{1}\right)$ TEM image (scale bar: $500 \mathrm{~nm}$ ) of LFM. (b) TEM image of $\kappa \mathrm{CH}$ formed by an interconnected network of a $\kappa$-carrageenan nanofibril $(\kappa \mathrm{Cnf})$, i.e., mesoscale units of $\kappa \mathrm{CH}$. (c) LFM-reinforced $\kappa \mathrm{CH}(1.1$ wt $\% \kappa \mathrm{CH}$ and $2.0 \mathrm{wt} \% \mathrm{LFM}$ ) showing how LFMs are finely woven by $\kappa \mathrm{Cnf}$ at the surface: $\left(c_{1}\right)$ lower magnification and $\left(c_{2}\right)$ higher magnification. Inset: zoom-in view of the LFM and $\kappa \mathrm{CH}$ connection showing $\kappa$ Cnf filaments at the surface (scale bar: $200 \mathrm{~nm}$ ). (d) Schematic of LFM-reinforced $\kappa \mathrm{CH}$ hydrogel and a visualized illustration of the stretchy behavior of the LFM $+\kappa \mathrm{CH}$ between the thumb and forefinger which is similar to the "beads-on-a-string" phenomenon often observed for a drop of saliva.

chains represent the nanofibrils. The visual image in Figure 1d visualizes the stretchy behavior of the $\mathrm{LFM}+\kappa \mathrm{CH}$ which is similar to the "the extensional filament-like behavior observed for a stretched drop of real human saliva".

The lubrication performance of the aforementioned lubricant compositions is first studied in rolling-sliding contacts on PDMS surfaces. Although the relevance of this conventional soft tribology test to study oral lubrication is of concern, PDMS as a model surface has been extensively used ${ }^{11}$ in oral tribology studies. Therefore, the tests using PDMS surfaces, as a classic framework to understand the lubrication behavior, offer cross-comparison of the obtained adsorption and friction results with the literature.
In classical lubrication theories for hard-on-hard contacts, typically four lubrication regimes have been defined in tribology science, ${ }^{12}$ which have been exploited in soft tribology. ${ }^{13,14}$ Although a distinct transition between two successive regimes is arduous, theoretical models and modified Stribeck curves (or friction vs a modified velocity parameter) have been proven useful in qualitative determination of regimes (Supporting Information, Figure S1). ${ }^{15,16}$ For ease of comparison in the tribo results, we use a product of lubricant viscosity and entrainment speed $(\eta U)$, which is wellestablished in tribological studies of soft surfaces. We also included the friction coefficient versus $\eta U$ for Newtonian fluids (as shown in Figure 2d) for comparison purposes. The $\eta U$ range in which the surfaces experience direct solid-solid contacts $\left(<\sim 5 \times 10^{-5} \mathrm{~Pa} \mathrm{~m}\right)$ and the friction coefficient remains relatively constant is referred to as the "boundary regime" hereafter. Upon increasing the entrainment speed, the friction coefficient reduces sharply where the lubricant film partially carries the load; that is the "mixed regime".

The friction coefficient reduces as the contribution of the direct contact between surfaces reaches its minimum (i.e., elasto-hydrodynamic regime) and the onset of a full-film lubrication can be observed. Following transition to the full fluid-film lubrication, hydrodynamic forces bring about a lubrication regime where the contact bodies are completely separated by a lubricant film and the lubricant viscosity renders an important role (i.e., hydrodynamic regime).

Figure 2a shows the friction curves for $2.0 \mathrm{wt} \% \mathrm{LFM}, 1.1 \mathrm{wt}$ $\% \kappa \mathrm{CH}$, and LFM-reinforced $\kappa \mathrm{CH}(\kappa \mathrm{CH}: \mathrm{LFM}$ at 0.07:1 wt/ vol., containing $1.1 \mathrm{wt} \% \kappa \mathrm{CH}$ and 2.0 wt $\%$ LFM) lubricant formulations in PDMS-PDMS tribo contacts. Friction curves for LFM and $\kappa \mathrm{CH}$ lubricants show nearly similar friction values across the speed range and lower values in comparison to those for the buffer. The friction curves for LFM and $\kappa \mathrm{CH}$ maintain such a decreasing trend in the friction coefficient values as speed increases regardless of the concentration changes, with relatively high friction coefficients at the relatively low entrainment speeds (i.e., $<0.01 \mathrm{~ms}^{-1}$ ) even at the highest concentration of original LFM and $\kappa \mathrm{CH}$ (see Supporting Information, Figure S2). On the other hand, LFM-reinforced $\kappa \mathrm{CH}$ shows an unprecedented superlubricity behavior with friction coefficient values as low as $1 \times 10^{-2}$ throughout the whole entrainment speed range investigated here (i.e., $1.31 \times$ $10^{-2}$ and $0.49 \times 10^{-2}$ at $0.005 \mathrm{~ms}^{-1}$ and $0.1 \mathrm{~ms}^{-1}$, respectively). Interestingly, the LFM-reinforced $\kappa \mathrm{CH}$ provides a superior lubricity as compared to real human saliva, demonstrating its potential as an effective biolubricant.

The mixture of untreated, i.e., nonmicrogelled, LF protein and $\kappa \mathrm{CH}$ (Supporting Information, Figure S3) does not show superlubricity. This indicates the importance of using LF in microgel form in order to allow a high degree of interaction with $\kappa \mathrm{CH}$. To the best of our knowledge, such a synergistic effect to yield super low friction coefficients has been particularly achieved through synthetic aqueous lubricants ${ }^{17,18}$ and is rarely reported for natural biopolymeric aqueous lubricants.

Figure $2 \mathrm{~b}$ shows the linear response of the elastic modulus for the hydrogel, microgels, and their mixture under shear in the frequency range of $0.1-10 \mathrm{~Hz}$ at a constant strain (see Supporting Information for determination of the linear regime, Figure S4). The elastic modulus of the LFM-reinforced $\kappa \mathrm{CH}$ $(13.26-36.63 \mathrm{~Pa})$ is one order of magnitude greater than that of the sole components at equivalent concentrations (0.48- 


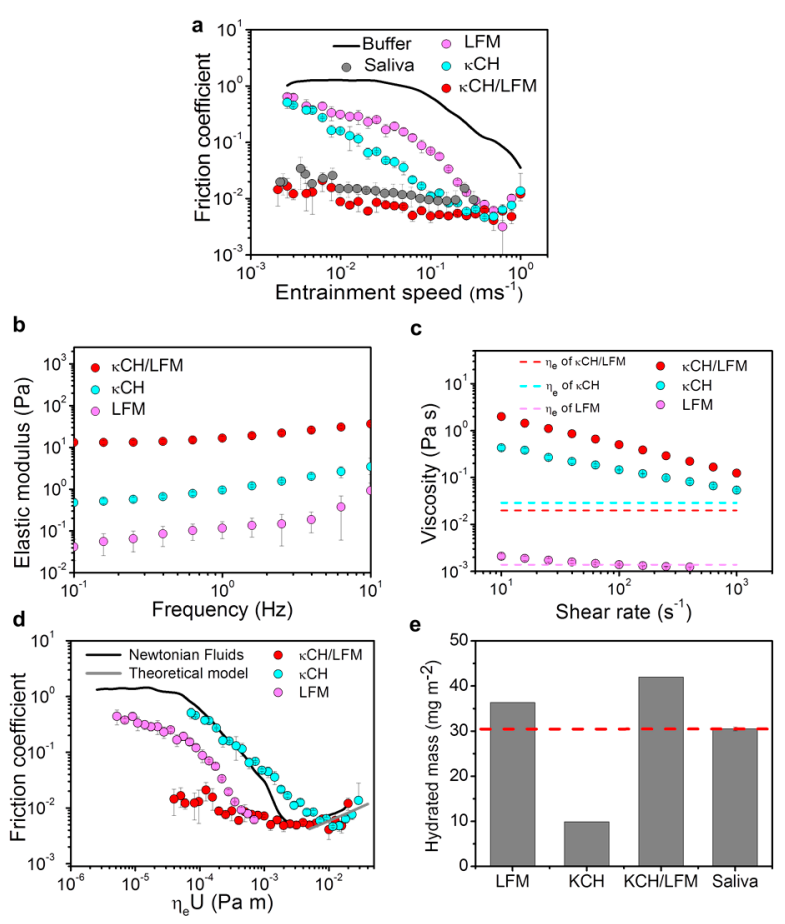

Figure 2. Tribological and rheological performances of the lubricants. $\kappa \mathrm{CH} / \mathrm{LFM}$ in panels represents LFM-reinforced $\kappa \mathrm{CH}$. (a) The friction results as a function of entrainment speed $(U)$ obtained for lubricants using an MTM tribometer and PDMS specimens. The $\kappa \mathrm{CH} / \mathrm{LFM}$ delivered a lubrication performance exceeding that of real human saliva. (b) Frequency dependence of the elastic modulus at a constant strain rate $(1.0 \%)$ for $1.1 \mathrm{wt} \% \kappa \mathrm{CH}, 2.0 \mathrm{wt} \% \mathrm{LFM}$, and LFM-reinforced $\kappa \mathrm{CH}$ containing $1.1 \mathrm{wt} \% \kappa \mathrm{CH}$ and $2.0 \mathrm{wt} \% \mathrm{LFM}$ (see details in Supporting Information, Figure S4). (c) Shear viscosity of LFM, $\kappa \mathrm{CH}$, and LFM-reinforced $\kappa \mathrm{CH}$ as a function of shear rate. $\eta_{\mathrm{e}}$ represents the effective tribological viscosity (see Supporting Information, Theory Section). (d) The friction coefficient results as a function of $\eta_{\mathrm{e}} U$. The gray solid line represents the estimated friction coefficient based on effective tribological viscosity (see Supporting Information, Theory Section). (e) The hydrated mass of adsorbed LFM, $\kappa \mathrm{CH}$, real human saliva, and LFM-reinforced $\kappa \mathrm{CH}$ onto PDMScoated sensors obtained by QCM-D and measured using the Voigt viscoelastic model applied to $3^{\text {rd }}-11^{\text {th }}$ overtones (raw data of frequency and dissipation shifts of $5^{\text {th }}$ overtone are available in Supporting Information, Figure S5). The red dotted line represents the adsorption level of saliva. Human saliva was collected from a healthy young female in the morning. The subject was refrained from eating and drinking for at least $2 \mathrm{~h}$ before saliva collection (Ethics number: MEEC 16-046, University of Leeds, UK); the saliva was diluted with $10 \mathrm{mM}$ HEPES at the ratio of $1: 1 \mathrm{w} / \mathrm{w}$ and centrifuged; and the supernatant was used for the tribology measurements. Values are presented as the means \pm SDs of nine readings on triplicate samples $(n=9 \times 3)$ except for QCM-D data $(n=3 \times 1)$.

3.48 Pa for $\kappa \mathrm{CH}, 0.04-0.93 \mathrm{~Pa}$ for LFM) throughout the whole frequency range measured here (Figure $2 \mathrm{~b}$ ). The elastic modulus for the LFM-reinforced $\kappa \mathrm{CH}$ is greater than that of sole LFM or $\kappa \mathrm{CH}$ at higher concentrations (see Supporting Information, Figures $\mathrm{S} 4 \mathrm{a}-\mathrm{S} 4 \mathrm{c}$ ). This indicates that the microgels behave as active fillers and hence reinforce the hydrogel's nanofibrillar network as observed in the TEM image. Such a synergistic effect raises a question toward the impact of viscous forces and surface adsorption on the lubrication performance of these hydrogels. The shear viscosity results for the same fluids are shown in Figure $2 \mathrm{c}$. Both $\kappa \mathrm{CH}$ and LFM-reinforced $\kappa \mathrm{CH}$ are shear thinning fluidsc. The LFM shows a relatively constant viscosity of $0.0016 \mathrm{~Pa}$ s which is at least two orders of magnitude lower than those of the other two fluids. The greater viscosity of LFM-reinforced $\kappa \mathrm{CH}$ as compared to $\kappa \mathrm{CH}$ implies the interactions between $\kappa \mathrm{CH}$ and LFM (i.e., reinforcement and filling action while retaining the fluidity).

The shear thinning behavior of LFM-reinforced $\kappa \mathrm{CH}$ and $\kappa \mathrm{CH}$ does not reach a plateau over the shear rate examined here. Therefore, the effective tribological viscosity $\left(\eta_{\mathrm{e}}\right)$ is estimated using friction values in the elasto-hydrodynamic lubrication regime and de Vicente et al.'s (2005) theoretical model (see Supporting Information, Equation S1). ${ }^{19}$ The obtained $\eta_{\mathrm{e}}$ values are shown using dashed lines, assuming the effective Newtonian behavior of fluids in the tribological limit. ${ }^{2}$

This brings us to evaluate the influence of viscous forces on the lubrication performance of the lubricant formulations by plotting the friction coefficient as a function of $\eta_{\mathrm{e}} U$ (Figure $2 \mathrm{~d}$ ). This graph sheds light on the influence of hydrodynamic viscous forces of a lubricant when compared to the performance of aqueous-based Newtonian fluid. Friction curve for $\kappa \mathrm{CH}$ overlapped with Newtonian fluid almost in all regimes. This indicates that the viscous force is the main contribution to the tribological performance of $\kappa \mathrm{CH}$. Friction values for LFM are considerably lower in comparison to the values for a Newtonian fluid irrespective of the speeds. This indicates the impact of viscous and hydration action of LFM on its boundary lubrication. On the other hand, LFMreinforced $\kappa \mathrm{CH}$ shows predominantly friction values which are dramatically lower than the friction coefficient values of other tested lubricants. Since the viscous behavior of LFMreinforced $\kappa \mathrm{CH}$ is similar to that of $\kappa \mathrm{CH}$ (Figure 2c), it is reasonable to attribute its super-low friction behavior to the hydration force which is bestowed on LFM-reinforced $\kappa \mathrm{CH}$ as a result of synergistic effects between LFM and $\kappa \mathrm{CH}$. As the entrainment speed increases, the friction curve of LFMreinforced $\kappa \mathrm{CH}$ overlaps with the graph for Newtonian fluid within the elasto-hydrodynamic lubrication regime, indicating that the lubrication of LFM-reinforced $\kappa \mathrm{CH}$ relies completely on the high shear rate viscosity of the solution in this regime.

The gray line in Figure $2 \mathrm{~d}$ presents the theoretical prediction of the friction coefficient in the hydrodynamic regime ${ }^{19}$ which shows a good agreement with the experimental results. The minimum film thickness $\left(h_{\mathrm{m}}\right)$ at the onset of the hydrodynamic regime (Supporting Information, Equation S2) for LFMreinforced $\kappa \mathrm{CH}, \kappa \mathrm{CH}$, and the Newtonian reference is estimated to be $1514.4,2118.2$, and $1762.5 \mathrm{~nm}$, respectively. ${ }^{19}$ The film thicknesses which are greater than the diameter of microgels (an average size below $200 \mathrm{~nm}$ shown in Figure 1a) facilitate interposition of the microgels at the contact interface, enabling fluids to act closely to a continuum.

As for the surface adsorption properties, the quartz crystal microbalance with dissipation monitoring (QCM-D) equipped with PDMS-coated crystals is used, and the associated results are shown in Figure 2e (see Supporting Information for frequency and dissipation shift data, Figure S5). The adsorption data for LFM and LFM-reinforced $\kappa \mathrm{CH}$ (hydrated mass of 36.0 and $40.0 \mathrm{mg} \mathrm{m}^{-2}$, respectively) show that their adsorption behavior on PDMS surfaces surpasses the adsorption activity of greater values of adsorbed saliva (32.0 $\mathrm{mg} \mathrm{m}^{-2}$ ). Despite $\kappa \mathrm{CH}$ having similar lubricating properties to LFM, absorption of the former is only about one third of the latter. Furthermore, $\kappa \mathrm{CH}$ only slightly enhances the surface adsorption properties of LFM-reinforced $\kappa \mathrm{CH}$, despite their 
significantly different lubrication performance. Thus, unlike reported for simple carbohydrate solutions, ${ }^{20,21}$ fluids presented here do not show exclusive correlation between friction and surface adsorption. This lack of correlation can be attributed to the static nature of QCM-D measurements which differs from shear-dominated tribo tests. ${ }^{22}$ Nevertheless, the high lubrication performance of LFM-reinforced $\kappa \mathrm{CH}$ results from the adsorption properties of LFM and the rheological properties of $\kappa \mathrm{CH}$, the latter helping the lubricant to remain at the contact interface even under tribological shear.

Further, we studied the lubrication performance of LFMreinforced $\kappa \mathrm{CH}$ compositions at different ratios to better understand the synergistic interactions between the lubricant components. For this purpose, the friction coefficient results as a function of the ratio of $\kappa \mathrm{CH}$ to LFM (wt/vol.) are presented in Figure $3 \mathrm{a}$ at $0.005 \mathrm{~ms}^{-1}$ and $0.1 \mathrm{~ms}^{-1}$. The full data for this
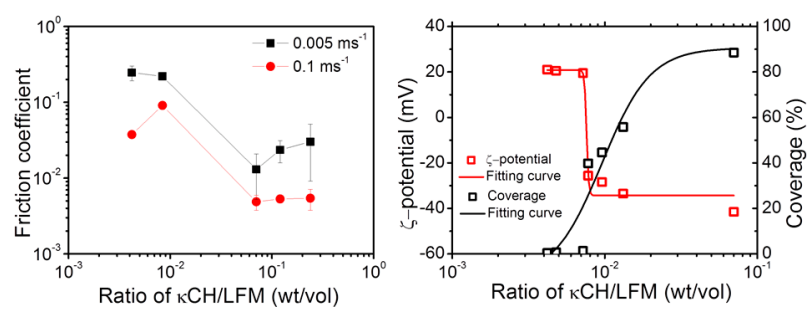

Figure 3. Influence of concentration ratio of $\kappa \mathrm{CH}$ to $\mathrm{LFM}$ on the tribological properties and $\zeta$-potential characteristics of the LFMreinforced $\kappa \mathrm{CH}$. (a) The friction coefficient results obtained at relatively low $\left(0.005 \mathrm{~ms}^{-1}\right)$ and high entrainment speeds $\left(0.1 \mathrm{~ms}^{-1}\right)$. (b) $\zeta$-Potential and surface coverage results, with the logistic fitting curve. Superlubricity (friction coefficient $\sim 10^{-2}$ ) is achieved only at $\kappa \mathrm{CH} / \mathrm{LFM}$ ratios of greater than 0.07:1 wt/vol $\kappa \mathrm{CH} / \mathrm{LFM}$. Values are presented as the mean \pm SDs of nine readings on triplicate samples $(n$ $=9 \times 3)$.

investigation can be found in the Supporting Information (Figure S6). As shown in Figure 3a, the friction coefficient at both speeds increased around one order of magnitude upon decrease of the relative concentration ratio to below $0.01 \mathrm{wt} /$ vol. This indicates that a sufficient amount of $\kappa \mathrm{CH}$ is required to achieve the observed superlubricity. To further elucidate this observation, $\zeta$-potential measurements are carried out on LFM-reinforced $\kappa \mathrm{CH}$ compositions. $\zeta$-Potential results as a function of the $\kappa \mathrm{CH}$ to LFM ratio are shown in Figure $3 \mathrm{~b}$. The $\zeta$-potential for the sole LFM and $\kappa \mathrm{CH}$ components is measured as +22.7 and $-46.3 \mathrm{mV}$ at $\mathrm{pH} 7.0$, respectively. Oppositely charged components propel mutual electrostatic attraction, resulting in a structure observed in TEM images (Figure 1c).

The $\zeta$-potential is sensitive to the $\kappa \mathrm{CH} / \mathrm{LFM}$ ratios, resulting in a decrease of electrophoretic mobility and consequently reduction in the net surface charge. As the $\kappa \mathrm{CH} / \mathrm{LFM}$ ratio increasees, the coverage of LFM by $\kappa \mathrm{Cnf}$ is enhancened. As a result of this, the LFM-reinforced $\kappa \mathrm{CH}$ undergoes a charge reversal process, at the shear plane, gradually from net positive towards net negative values. The coverage curve, calculated from $\zeta$-potential results (Supporting Information, Equation S3), ${ }^{23}$ reaches a plateau of around $90 \%$ at a ratio of 0.07:1 wt:vol. The friction coefficient curve plateaued out approximately at the same ratio (Figure 3a). These results are in agreement with TEM observations for LFM-reinforced $\kappa \mathrm{CH}$ at a ratio of 0.07:1 wt:vol (Figure 3c). These corroborate that an adequate level of coverage of LFM by the $\kappa \mathrm{Cnf}$ is required for LFM-reinforced $\kappa \mathrm{CH}$ to deliver superlubricity.

Finally, the efficacy of the developed lubricant formulation on a tongue-emulated surface is assessed using the method developed by Andablo-Reyes et al. ${ }^{24}$ A set of experiments are performed under orally relevant conditions with respect to speed, pressure, and surface properties. Briefly, a soft Ecoflex 00-30 was used to create silicone surfaces from a 3D-printed mold, with Young's modulus of $\sim 120 \mathrm{kPa}$, latter being considerably closer to the modulus of the tongue as compared to PDMS. The mold contains papillae-shaped features with appropriate size and spatial distribution of fungiform and filiform papillae, mimicking those present on the real human tongue. Span 80 (0.5 wt \%) was used to enhance the wettability of the Ecoflex 00-30 in some experiments. The water contact angle for the surfaces containing Span 80 was measured to be $76.0^{\circ} \pm 2.0,^{24}$ resembling the wettability of tongue surfaces with some degree of adsorbed saliva on top. More details with reference to this testing approach can be found in a recent work by the authors. ${ }^{24}$ Figure 4 shows friction curves for all
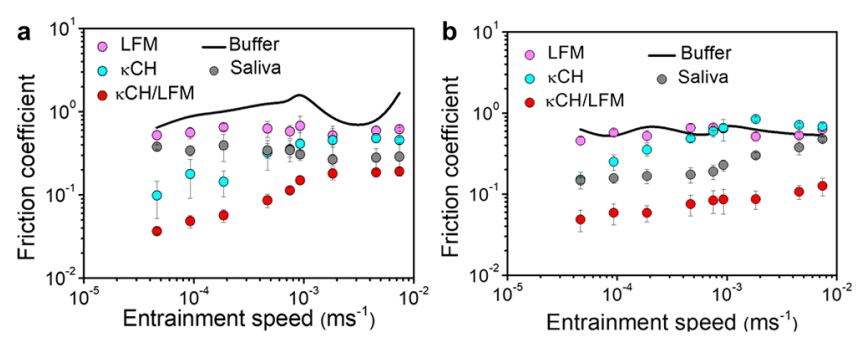

Figure 4. Tribological performances of the lubricants obtained using tongue-mimicking tribological surfaces. LFM-reinforced $\kappa \mathrm{CH}$ demonstrates the lowest friction values as compared to buffer, saliva, $\kappa \mathrm{CH}$, or LFM. Friction results obtained using soft textured (a) Ecoflex 0030 (hydrophobic) and (b) Ecoflex 00-30 + Span80 (hydrophilic) surfaces. Human saliva was collected from a healthy young female on the morning of the testing day. The subject was refrained from eating and drinking for at least $2 \mathrm{~h}$ before saliva collection (Ethics number: MEEC 16-046, University of Leeds, UK). The saliva was diluted with $10 \mathrm{mM}$ HEPES at the ratio of $1: 1 \mathrm{w} / \mathrm{w}$ and centrifuged, and the supernatant was used for the tribology measurements. Values are presented as the means \pm SDs of nine readings on triplicate samples $(n=9 \times 3)$.

lubricants used in this study. Panels a and b present the results for soft and textured Ecoflex 00-30 (hydrophobic) and soft Ecoflex 00-30 + Span80 (hydrophilic) materials, respectively. LFM shows poor lubrication behavior, with a slight reduction in the friction coefficient when compared to the buffer. $\kappa \mathrm{CH}$ provides relatively better lubricity as compared to buffer and LFM for both surfaces at speeds below $10^{-3} \mathrm{~m} / \mathrm{s}$. The lubrication performance of saliva is found to be dependent on the wettability behavior of the surfaces, particularly at speeds below $10^{-3} \mathrm{~m} / \mathrm{s}$. For saliva, lower friction coefficients are observed on Ecoflex 00-30 + Span80 surfaces which surpass the lubricity of both LFM and $\kappa \mathrm{CH}$.

Results show that tribo-test conditions (contact pressure, contact geometry, and sample topography and chemistry) used with tongue-mimicking surfaces and surface properties have a large impact on the performance of the fluids (compare Figure 4 to Figure 2a). Interestingly and in agreement with the MTM results (Figure 2a), LFM-reinforced $\kappa \mathrm{CH}$ outperformed the other fluids within the whole speed range measured here, with friction coefficient values an order of magnitude lower with 
respect to the values for the buffer. These results prove the superlubricity of LFM-reinforced $\kappa \mathrm{CH}$ for soft silicon surfaces that emulated the stiffness and topography of the human tongue with the Ecoflex 00-30 representing extreme dry mouth conditions and the modified material (Ecoflex 00-30+ Span80), representing a dry tongue with some residual saliva.

In summary, we have developed a new sophisticated nonlipidic biolubricant composed of submicron-sized lactoferrin microgels dispersed in $\kappa$-carrageenan hydrogel. The biolubricant possesses superlubricity characteristics which exceed the lubricity of real human saliva in different oral mimicking conditions. The excellent lubrication performance of this microgel-reinforced hydrogel is attributed to the synergistic effects between LFM and $\kappa \mathrm{CH}$. The synergistic effects impart superlubricity to the lubricant which is facilitated through viscous forces and surface adsorption. ${ }^{25}$ This new hydrogel has significant potential for applications in oral care products where lubrication without lipid content is desired. For instance, dry mouth syndrome or xerostomia ${ }^{26}$ poses a limitation to the lubrication of oral surfaces in the absence of natural saliva. In addition, intake of extra lipid for lubrication can be undesirable for these patients especially for the elderly population, where this condition is prevalent. Thus, the development of bio-inspired lubricants as an alternative to saliva, such as our LFM-reinforced $\kappa \mathrm{CH}$, is a high priority. Additionally, the present formulation can be potentially used to replicate the lubricating properties of fat content in food products, providing the possibility of decreasing caloric content, without sacrificing sensory-related attributes. ${ }^{27}$

\section{ASSOCIATED CONTENT}

\section{SI Supporting Information}

The Supporting Information is available free of charge at https://pubs.acs.org/doi/10.1021/acsmacrolett.0c00689.

Experimental section, theory section, and supplementary figures (PDF)

\section{AUTHOR INFORMATION}

\section{Corresponding Author}

Anwesha Sarkar - Food Colloids and Bioprocessing Group, School of Food Science and Nutrition, University of Leeds, Leeds LS2 9JT, U.K.; (1) orcid.org/0000-0003-1742-2122; Email: a.sarkar@leeds.ac.uk

\section{Authors}

Jing Hu - Food Colloids and Bioprocessing Group, School of Food Science and Nutrition, University of Leeds, Leeds LS2 9JT, U.K.

Efren Andablo-Reyes - Food Colloids and Bioprocessing Group, School of Food Science and Nutrition, University of Leeds, Leeds LS2 9JT, U.K.

Siavash Soltanahmadi - Food Colloids and Bioprocessing Group, School of Food Science and Nutrition, University of Leeds, Leeds LS2 9JT, U.K.; (1) orcid.org/0000-0002-69094776

Complete contact information is available at:

https://pubs.acs.org/10.1021/acsmacrolett.0c00689

\section{Author Contributions}

The manuscript was written through contributions of all authors.

\section{Funding}

The European Research Council (ERC) under the European Union's Horizon 2020 research and innovation programme (Grant agreement $\mathrm{n}^{\circ} 757993$ and 890644).

\section{Notes}

The authors declare no competing financial interest.

The data presented in this article will be openly available from the University of Leeds Data Repository https://doi.org/10. $5518 / 933$.

\section{REFERENCES}

(1) Lee, S.; Spencer, N. D. Sweet, Hairy, Soft, and Slippery. Science 2008, 319, 575 .

(2) Andablo-Reyes, E.; Yerani, D.; Fu, M.; Liamas, E.; Connell, S.; Torres, O.; Sarkar, A. Microgels as Viscosity Modifiers Influence Lubrication Performance of Continuum. Soft Matter 2019, 15, 96149624.

(3) Deshmukh, O. S.; van den Ende, D.; Stuart, M. C.; Mugele, F.; Duits, M. H. Hard and Soft Colloids at Fluid Interfaces: Adsorption, Interactions, Assembly \& Rheology. Adv. Colloid Interface Sci. 2015, 222, 215-27.

(4) Hong, S.; Sycks, D.; Chan, H. F.; Lin, S.; Lopez, G. P.; Guilak, F.; Leong, K. W.; Zhao, X. 3D Printing of Highly Stretchable and Tough Hydrogels into Complex, Cellularized Structures. Adv. Mater. 2015, 27, 4035-40.

(5) Lee, K. Y.; Mooney, D. J. Hydrogels for Tissue Engineering. Chem. Rev. 2001, 101, 1869-1880.

(6) Sun, J. Y.; Zhao, X.; Illeperuma, W. R.; Chaudhuri, O.; Oh, K. H.; Mooney, D. J.; Vlassak, J. J.; Suo, Z. Highly Stretchable and Tough Hydrogels. Nature 2012, 489, 133-136.

(7) Liu, G.; Liu, Z.; Li, N.; Wang, X.; Zhou, F.; Liu, W. Hairy Polyelectrolyte Brushes-Grafted Thermosensitive Microgels as Artificial Synovial Fluid for Simultaneous Biomimetic Lubrication and Arthritis Treatment. ACS Appl. Mater. Interfaces 2014, 6, 2045220463.

(8) Sarkar, A.; Hu, J.; Andablo-Reyes, E. Formulation, GB Patent 2007546.1, May, 2020.

(9) Hermansson, A. M.; Eriksson, E.; Jordansson, E. Effects of Potassium, Sodium and Calcium on the Microstructure and Rheological Behaviour of kappa-Carrageenan Gels. Carbohydr. Polym. 1991, 16, 297-320.

(10) Liu, J.; Zhan, X.; Wan, J.; Wang, Y.; Wang, C. Review for Carrageenan-based Pharmaceutical Biomaterials: Favourable Physical Features versus Adverse Biological Effects. Carbohydr. Polym. 2015, $121,27-36$.

(11) Torres, O.; Andablo-Reyes, E.; Murray, B. S.; Sarkar, A. Emulsion Microgel Particles as High-Performance Bio-Lubricants. ACS Appl. Mater. Interfaces 2018, 10, 26893-26905.

(12) Hutchings, I., Shipway, P. Tribology: Friction and Wear of Engineering Materials, 1st ed.; Butterworth-Heinemann: Cambridge, 2017; p 327.

(13) Bongaerts, J. H. H.; Fourtouni, K.; Stokes, J.R. Soft-Tribology: Lubrication in a Compliant PDMS-PDMS Contact Tribol. Tribol. Int. 2007, 40, 1531-1542.

(14) de Vicente, J.; Stokes, J. R.; Spikes, H. A. Rolling and Sliding Friction in Compliant, Lubricated Contact. Proc. Inst. Mech. Eng., Part J 2006, 220, 55-63.

(15) Dowson, D. Thin Films in Tribology. Tribol. Ser. 1993, 25, 312.

(16) Caravia, L.; Dowson, D.; Fisher, J.; Corkhill, P.H.; Tighe, B.J. Friction and Mixed Lubrication in Soft Layer Contacts. Tribol. Ser. 1993, 25, 529-534.

(17) Qu, J.; Barnhill, W. C.; Luo, H.; Meyer, H. M.; Leonard, D. N.; Landauer, A. K.; Kheireddin, B.; Gao, H.; Papke, B. L.; Dai, S. Synergistic Effects Between Phosphonium-Alkylphosphate Ionic Liquids and Zinc Dialkyldithiophosphate (ZDDP) as Lubricant Additives. Adv. Mater. 2015, 27, 4767-4774. 
(18) Sun, Z.; Feeney, E.; Guan, Y.; Cook, S. G.; Gourdon, D.; Bonassar, L. J.; Putnam, D. Boundary Mode Lubrication of Articular Cartilage with a Biomimetic Diblock Copolymer. Proc. Natl. Acad. Sci. U. S. A. 2019, 116, 12437.

(19) de Vicente, J.; Stokes, J. R.; Spikes, H. A. The Frictional Properties of Newtonian Fluids in Rolling-Sliding Soft-EHL Contact. Tribol. Lett. 2005, 20, 273-286.

(20) Stokes, J. R.; Macakova, L.; Chojnicka-Paszun, A.; de Kruif, C. G.; de Jongh, H. H. J. Lubrication, Adsorption, and Rheology of Aqueous Polysaccharide Solutions. Langmuir 2011, 27, 3474-3484.

(21) Zhang, J.; Meng, Y. Boundary lubrication by adsorption film. Friction 2015, 3, 115-147.

(22) Majd, SE.; Kuijer, R.; Köwitsch, A.; Groth, T.; Schmidt, TA.; Sharma, PK Both Hyaluronan and Collagen Type II Keep Proteoglycan 4 (Lubricin) at the Cartilage Surface in a Condition that Provides Low Friction during Boundary Lubrication. Langmuir 2014, 30, 14566-14572.

(23) Pallandre, S.; Decker, E. A.; McClements, D. J. Improvement of Stability of Oil-in-Water Emulsions Containing Caseinate-Coated Droplets by Addition of Sodium Alginate. J. Food Sci. 2007, 72, E518-E524.

(24) Andablo-Reyes, E.; Bryant, M.; Neville, A.; Hyde, P.; Sarkar, R.; Francis, M.; Sarkar, A. 3D Biomimetic Tongue-Emulating Surfaces for Tribological Applications. ACS Appl. Mater. Interfaces 2020, 12, 49371-49385.

(25) Xu, F.; Liamas, E.; Bryant, M.; Adedeji, A. F.; Andablo-Reyes, E.; Castronovo, M.; Ettelaie, R.; Charpentier, T. V. J.; Sarkar, A. A Self-Assembled Binary Protein Model Explains High-Performance Salivary Lubrication from Macro to Nanoscale. Adv. Mater. Interfaces 2020, 7, 1901549.

(26) Porter, S. R.; Scully, C.; Hegarty, A. M. An update of the Etiology and Management of Xerostomia. Oral Surg Oral Med. Oral Pathol Oral Radiol Endod 2004, 97, 28-46.

(27) Sarkar, A.; Krop, E. M. Marrying Oral Tribology to Sensory Perception: a Systematic Review. Curr. Opin. Food Sci. 2019, 27, 6473. 